\title{
Le juge pénal congolais, civil ou militaire, n'a pas le droit, ni de blâmer le ministère public, ni de lui faire des observations et encore moins, de lui donner des injonctions
}

\author{
Par MAJAMBO KAMENGA David Gaël ${ }^{*}$ et KISHIBA MALIFWA Clémence**
}

\section{INTRODUCTION}

L'exigence de la célébrité de la procédure est une garantie essentielle d'un procès équitable, le législateur congolais en a eu telle conscience qu'il a, beaucoup plus, d'ailleurs, dans le Code Judiciaire Militaire que dans le Code de procédure Pénale, institué plusieurs dispositions légales qui rendent possible la réalisation de cet objet dans chaque procès pénal. $\mathrm{Au}$ nombre desdites dispositions, figurent, sans nul doute, les articles 67 et 74, alinéa 6, du premier code cité, d'une part, et les articles 219, 249 et 253, alinéas 3 et 4, du second.

Mais, du point de vue technique, il s'indique de faire observer que les deux codes de procédure pénale prévoient la possibilité de supplément d'instruction, selon le cas, dans deux cas, de figure : le premier, au visa des articles 67 ou 219 CJM, avant l'ouverture des débats, c.à.d., pendant la procédure antérieure à l'audience ou aux débats tandis que, la seconde, sur fond des articles 74, alinéa 6 CPP et 253, alinéa 3et 4, CJM, au cours de ceux-ci.

$\mathrm{Eu}$ égard à l'étendue trop vaste du sujet, la présente étude, plus modeste, s'attèlera uniquement à l'examen de l'article 219 CJM et délaissera celui de l'article 67 CPP, qui est son pendant en droit procédural ordinaire, au motif que cette dernière disposition, témoignait le professeur Nicolas-Abel Bayona ${ }^{1}$, n'a jamais, à sa connaissance, fait l'objet d'application, ce qui est loin d'en être le cas s'agissant de l'article 219 CJM dont l'interprétation suscite de très vives controverses autour de l'identification de l'autorité judiciaire destinataire ou légalement chargée de l'exécution de l'injonction du président de la juridiction militaire dont question dans cette disposition légale précitée.

En effet, si certaines opinions retiennent - à tort, il nous semble - que le président d'une juridiction (en fait, d'une composition de celle-ci) est fondée de donner des injonctions au ministère public en vue de la complétude d'une instruction dans des cas prévus par la loi, nous avons toujours, pour notre part, estimons le contraire et nous opposons farouchement à l'opinion de ceux-là en raison de l'indépendance réciproque du ministère public à l'égard des juges (article $219 \mathrm{CJM}$ ) ou des juridictions (Article 253, alinéas 3 et 4, CJM). C'est pourquoi, pour une meilleure intelligence de cet article 219 CJM, nous jugeons bon

* Chef de Travaux à la Faculté de Droit de l'Université de Lubumbashi.

** Assistante à la Faculté de Droit de 1'Université de Lubumbashi

1 BAYONA (Nicolas-Abel), Réflexions sur la portée de l'article 67 CPP, in Le Plumitif, Collection B. Kingudi, $\mathrm{n}^{\circ} 4$, Lubumbashi, 2017, P.51 (qui a repris la version originaire de 1'article de la Revue Congolaise de droit). 
de rappeler, à l'entame de notre réflexion, sa formulation, ainsi que celle de l'article 221 CJM qui est sa suite logique. Ils sont, en effet, libellés, l'un et l'autre, comme suit :

«Le juge militaire saisi peut, si l'instruction préparatoire lui semble incomplète ou si des éléments nouveaux sont révélés depuis sa clôture, ordonner tous actes d'instruction qu'il estime utiles. Il est procédé à ces actes conformément aux dispositions relatives à l'instruction préparatoire par l'auditeur militaire près cette juridiction. "》. (Article 219 CJM)

Tandis que, le second :

"Les procès-verbaux et les autres pièces ou documents réunis au cours du supplément d'instruction sont déposés au greffe de la juridiction et versés au dossier de la procédure. Ils sont mis à la disposition du Ministère Public et du conseil du prévenu qui sont avisés de leur dépôt par les soins du greffier. »(Article 221 CJM)

En vue de cette démonstration, nous nous appuierons sur des raisons tirées de la loi (Section 1), de celles tirées de la doctrine (Section 2) et de la jurisprudence (Section 3) et, enfin, de manière plus intéressante encore, sur celles liées à d'importantes considérations constitutionnelles (Section 4)...

\section{A. LES RAISONS TIREES DE LA LOI}

Les raisons que nous tirons de la loi tiennent compte non seulement des précédents historiques des actuels articles 219 et 221 CJM que sont les articles 185 du Code de Justice militaire français porté par la loi n $65-542$ du 8 juillet 1965 (I) et 213 de l'OL n $72-006$ du 25 septembre 1972 portant institution d'un Code de Justice Militaire (II), mais aussi de l'analyse détaillée de cet article 219 CJM actuel. (III).

\section{L'ARTICLE 185 CJM FRANÇAIS}

En vue de capter le sens d'un texte, la doctrine recommande de recourir notamment aux précédents historiques $^{2}$ ou aux travaux préparatoires dudit texte. Or, le Code de Justice Militaire porté par l'OL du 25 septembre 1972 ne comportait pas, en raison des modalités constitutionnelles $^{3}$ de sa confection, des travaux préparatoires, ce qui justifie, pour une meilleure intelligence de cet article 219 CJM, notre recours à l'article 185 CJM français, étant, d'ailleurs, donné que ce dernier Code avait plus que fortement inspiré notre Code de Justice Militaire de septembre 1972.

Situé, d'ailleurs, au chapitre relatif à la procédure antérieure à l'audience, cet article 185 CJM Français était libellé comme ci-après :

2 VITU Andre, Procédure Pénale, PUF, Paris, 1957, P. 5 et suivants.

3 Les OL étaient, sous la Deuxième République, des textes de nature législative que prenait le Chef de L'Etat en cas d'urgence et de vacances parlementaires. 
"Le Président, si l'instruction lui semble incomplète ou si des éléments nouveaux ont été révélés depuis la clôture de l'instruction ou la traduction directe peut ordonner tous actes d'instructions qu'il estime utile ».

"Il est procédé conformément à l'instruction préparatoire, soit par un magistrat assesseur ou le juge d'instruction militaire près le tribunal qu'il délègue à cette fin "».

"Les procès-verbaux et les autres pièces ou documents réunis au cours du supplément d'information sont déposés au greffe et joints au dossier de la procédure ».

"Ils sont mis à la disposition du ministère Public et du conseil du prévenu qui sont avisés de leur dépôt par les soins du greffier».

"Le commissaire du gouvernement peut, à tout moment, requérir communication de la procédure à charge de rendre les pièces dans les vingt-quatre heures. »

Quelques observations s'imposent à ce niveau :

- D'abord, la formation de cette disposition légale traduit fidèlement l'esprit d'Independence mutuelle et réciproque entre le parquet et les juges dans lequel le législateur avait conçu et voulu ladite disposition;

- Ensuite, en insérant une virgule à l'alinéa 2 de cette disposition, précisément après le mot instruction, le législateur semble avoir précisé sa propre pensée en ce que l'une ou l'autre autorité qu'il désigne comme celui devant diligenter ce supplément d'instruction le ferait selon les règles de l'instruction préparatoire étant entendu que le même législateur n'avait pas prévu des règles ou une procédure spéciale devant gouverner l'instruction que doit mener l'une ou l'autre de ces autorités juridictionnelles dans le cadre de ce supplément d'information.

- Enfin, que les alinéas 3 et 4 de cette disposition légale démontrent à suffisance que le ministère Public est étranger à l'accomplissement de ce supplément d'information du triple fait que celui, après son accomplissement, doit être mis à sa disposition, qu'il doit préalablement être avisés de son dépôt et qu'il lui est donné la possibilité de requérir, à tout moment, communication de telle ou telle autre pièce réunie au cours de ce supplément d'information, quitte à devoir la rendre dans les 24 heures suivant sa réception.

\section{L'ARTICLE 213 CJM ZAIROIS}

L'article 213 CJM du 25 septembre 1972 fut une copie conforme de l'article 185 CJMF, à regarder de près leur formulation. Cet article 213 CJM était, d'ailleurs, pour mémoire, formulé comme suit : 
"Le magistrat militaire, membre du conseil de guerre 4 ou le président ${ }^{5}$, si l'instruction lui semble incomplète ou si des éléments nouveaux ont été révélés depuis la clôture de l'instruction ou la traduction directe peut ordonner tous actes d'instruction ${ }^{6}$ qu'il estime utile ».

"Il est procédé conformément à l'instruction préparatoire, soit par le magistrat instructeur, soit par l'auditeur militaire près cette juridiction ».

"Les procès-verbaux et les autres pièces ou documents réunis au cours du supplément d'information sont déposés au greffe et joints au dossier de la procédure ».

"Ils sont mis à la disposition du ministère Public et du conseil du prévenu qui sont avisés de leur dépôt par les soins du greffier $»$.

"L'auditeur militaire peut, à tout moment, requérir communication de la procédure ».

Le Code de Justice Militaire Zaïrois de septembre 1972 est une copie quelque peu grossière $\mathrm{du}$ Code de justice Militaire français, du reste, très mal tropicalisée. La preuve, parmi tant d'autre, est, à titre illustratif, la reproduction de cet article 213 CJM qui semble comprendre, et transposer incorrectement l'institution de juge d'instruction par celle de « magistrat instructeur $»$ ou encore confier à l'auditeur militaire les pouvoirs que cet article 185 CJM français a, dans son code et conformément à une logique judiciaire très cohérente, reconnu, tantôt au président de la juridiction, au juge d'instruction ou au magistrat assesseur (article 185, alinéa $1^{\mathrm{er}}, \mathrm{CJMF}$ ), tantôt au commissaire du Gouvernement ou ministère public (article 185, alinéa 5 CJMF).

Mais, en gros, cette formulation de l'article 213 CJM ne s'est pas beaucoup, par rapport à notre sujet, écarté de la formulation du Code Justice Militaire français cité ci-haut, voire que en dépit de l'incise après une virgule de l'incise «soit, par le magistrat instructeur, soit par l'auditeur militaire, les alinéas 3, 4 et 5 de cet article 213 CJM restaient têtus pour confirmer, dans la logique de la présentation de cette disposition légale, que « l'autorité » chargée de l'exécution du supplément d'instruction sur injonction du président de la composition du tribunal ne pouvait être le ministère public puisqu'il ne se conçoit pas, si c'est dernier est celui qui doit accomplir ces devoirs d'instruction supplémentaires, que le greffier ait encore à mettre le dossier constitué au terme de cette instruction supplémentaire à la disposition du même Ministère Public et aviser celui-ci de son dépôt, encore moins qu'il soit prévu qu'il peut demander à tout moment communication du dossier.

4 Cette expression désignait le magistrat militaire professionnel, membre permanent des juridictions militaires de l'époque, du reste, dénommées conseils de guerre.

5 Ces conseils de guerre n'étaient pas forcément présidés par un magistrat de carrière.

6 Il se posait aussi un problème de formulation de ce texte depuis le droit français. En effet, le supplément d'instruction ne pouvait s'entendre que lorsque la juridiction militaire était saisie par décision de renvoi. 


\section{LES RAISONS TIREES DE L'ANALYSE DES ART. 219 ET CJM}

Le grand reproche qui mérite d'être adressé certains auteurs et praticiens s'agissant relativement à la portée et à l'intelligence de cet article 219, alinéa 2, CJM est leur interprétation mécanique et isolée de cette disposition alors qu'une telle entreprise, si elle repose réellement sur un raisonnement juridique correct et sur le bon sens, nécessite la prise en compte du contexte et des précédents historiques du texte à interpréter. En effet, nul n'ignore d'ailleurs, à cet effet, que l'interprétation d'un texte sans son contexte n'est qu'un pur prétexte. $^{7}$

Il en suit que la démarche consistant à interpréter mot à mot de cet alinéa 2 de l'article 219 CJM est tout autant spécieuse qu'incompatible avec les dispositions de l'article 211 CJM en ce que conséquent avec son histoire, il dissipe tout doute possible sur la vraie interprétation ou intelligence de l'article 219, alinéa 2, CJM précisément lorsqu'il indique non seulement que tous les procès-verbaux et les autres pièces ou documents réunis au cours du supplément d'information seront déposés au greffe de la juridiction concernée et joints au dossier de la procédure, mais aussi qu'il devront être mis à la disposition du Ministère Public et du conseil du prévenu, lesquels devront être avisés de leur dépôt par les soins du greffier.

Or, en ayant même le plus petit égard à la petite histoire de cette disposition et en se référant au bon sens, un raisonnement juridique moyen, s'appuyant sur une interprétation de l'ensemble des dispositions qui instituent la procédure antérieure à l'audience, ne pourrait éprouver des difficultés à réaliser que l'autorité judiciaire d'exécution de ce que peut éventuellement ordonner le président d'une composition du tribunal ne peut en aucun cas être le Ministère Public.

Et même alors, d'analyse syntactique de l'alinéa 2 de cet article 219 CJM laisse toujours peser les doutes au sujet du vœu réel du législateur à ce sujet. Il aurait fait attention à certaines exigences de la construction phraséologique et à la place des virgules ou des ponctuations dans une telle entreprise. Aussi, cet alinéa 2 de l'article 219 CJM n'aurait pas été formulé comme suit :

"Il est procédé à ces actes, conformément aux dispositions relatives à l'instruction préparatoire, par l'auditeur militaire près cette juridiction ».

Plutôt, probablement comme ci-après, en y ajoutant deux virgules à la même formulation originaire :

Il est procédé à ces actes, conformément aux dispositions relatives à l'instruction préparatoire, par l'auditeur militaire près cette juridiction. »

7 Autrement dit, au terme instruction préparatoire. Mais qui, lorsqu'elle était saisie par traduction directe, au terme d'une simple enquête? L'observation vaut son pesant d'or en ce que les deux CJM français et zaïrois dénommait la procédure devant les officiers de police judiciaire de « procédure d'enquête ». 
Ou encore, en ne changeant que la place des mots :

Il est procédé à ces actes par l'auditeur militaire près cette juridiction conformément aux dispositions relatives à l'instruction préparatoire.

\section{B. LES RAISONS TIREES DE LA DOCTRINE}

La doctrine ${ }^{8}$ française, (I), belge (II) et congolaise (III) nous seront d'un grand secours dans notre entreprise de démonstration.

\section{EN DROIT FRANÇAIS}

Relativement à l'indépendance du ministère Public à l'égard des juridictions, le Professeur André Vitu avait déclaré, à l'occasion du colloque organisé sous l'égide de la fédération Cohérente Europe et celui de la faculté de droit de Nancy, sous la Direction de Françaos Jacquot :

"Cette indépendance s'exprime, d'abord, dans ses rapports avec les juridictions à l'égard: les tribunaux ne peuvent ni obliger un parquet à agir, ni à exercer des poursuites, ni à prendre des réquisitions, et il ne peut pas non plus blâmer ce que ferait le ministère public. La Cour de cassation serait très stricte à cet égard si l'on se permettait d'oublier cela. "

Le magistrat du ministère public jouit d'une indépendance absolue vis à vis des juridictions en ce sens que le président de la cour ou du tribunal ne peut lui donner d'ordre, ni lui adresser des blâmes ou des injonctions

\section{EN DROIT BELGE}

Dans le même sens, voici, d'ailleurs, ce que, en droit judiciaire belge, écrivirent Henri-D Bosly, Damien Vandermeersh et Marie-Aude Beernaeert ${ }^{9}$ :

«Les juridictions et le ministère public jouissent d'une indépendance l'un vis-à-vis des de l'autre. Elle est consacrée par l'article 151 de la constitution. Nonobstant son statut hybride d'agent de l'exécutif et d'organe judiciaire, le ministère public se caractérise par son indépendance envers les cours et tribunaux et, dans une moindre mesure, envers le gouvernement. Il n'est ni l'assesseur, ni l'auxiliaire du juge et les juridictions répressives n'ont pas le pouvoir de lui adresser des injonctions, ni de censurer son action. Réciproquement, le ministère public n'a pas à s'immiscer dans la fonction du juger. »

9 BOSLY HENRI-D. et alii, Droit de la Procédure pénale, Ed. de la Charte, BRUGGE, 2008, p. 22. 
Et, les même ${ }^{10}$, plus loin :

" Ainsi les juridictions répressives n'ont pas le pouvoir d'adresser les injonctions au ministère public : ni de censurer son action. Le juge pénal ne peut notamment pas apprécier la décision du procureur d'entamer les poursuites ou lui donner des ordres à cet égard."

Il en va de même du Procureur Générale Cedrick Visart de Bocarme ${ }^{11}$, dans son discours intitulé le statut du Ministère Public, Conseil Supérieur de la Magistrature, présenté à Rome, en date du 4 juillet 2011.

"L'indépendance du Ministère Public se manifeste à l'égard des cours et tribunaux. A cet égard, les juridictions répressives n'ont pas le pouvoir d'adresser des injonctions au Ministère Public ni de censurer son action. Le juge pénal ne peut notamment pas apprécier les décisions du Procureur du Roi d'entamer les poursuites ou de lui donner des ordres à cet égard."

\section{EN DROIT CONGOLAIS}

Dans le même registre et plus près de nous, Antoine Rubbens faisait observer à cet égard ce qui suit :

"Les tribunaux n'ont pas d'injonctions à donner aux membres du ministère public; sous peine de déni de justice, ils doivent répondre à leurs réquisitions; ils ne peuvent, ni verbalement, ni dans le motif d'un jugement, censurer les actes du ministère public sans commettre un excès de pouvoir. ».

De même, le Doyen Nicolas-Abel Bayona Ba Meya Muna Kinvimba ${ }^{12}$ et le professeur Luzolo Bambi Lessa ont écrit, eux aussi, à ce sujet :

Les officiers du Ministère Public sont d'abord indépendants des juges. Ceux-ci ne peuvent se permettre de blâmer les magistrats du parquet dans leurs décisions ou dans leurs paroles. Ils ne peuvent pas non plus donner des injonctions de poursuites aux magistrats du parquet (...). En effet, le ministère public est le seul juge de l'exercice de l'action publique, estimant qu'à son avis, le dossier reste complet. Si le tribunal saisi constate des lacunes, il lui appartient de compléter l'instruction. ».

10 BOSLY HENRI-D. et alii, op cit, pp. 172-173.

$11 \mathrm{http} / / / \mathrm{www} . c o e$. int $/ \mathrm{t} / \mathrm{dghl} /$ cooperation/CCPE

12 LUZOLO BAMBI LESSA et BAYONA BA MEYA, Manuel de Procédure Pénale, PUC, Kinshasa, 2011, p. 206. 


\section{LES RAISONS TIREES DE LA JURISPRUDENCE DE LA CSJ}

Il y a lieu de citer deux arrêts importants de la Cour Suprême de Justice ${ }^{13}$ : le premier est le fameux « arrêt Gécomines » très largement connu de la plupart des juristes en raison des commentaires, dans tous les sens, qu'il avait suscités depuis l'époque à nos jours, tandis que le second, évidemment moins connu que le premier, l'arrêt Yav Mukuta Kabwe ${ }^{14}$ contre Bindscedler dans lequel cette même haute Cour avait réaffirmé le principe par elle énoncé dans l'arrêt précédent.

Il est urgent, à ce niveau, de faire observer que tous les juges militaires, même ceux de la Haute Cour Militaire, sont tenus au respect ou l'observance stricte de la jurisprudence de la Cour de cassation désormais compétente pour connaitre de tous pouvoirs en cassation dirigés contre les décisions de toutes les juridictions de l'ordre judiciaire dont toutes les juridictions militaires font parties.

\section{LE TRES FAMEUX ARRET GECOMINES}

Le principe de l'indépendance réciproque du juge et du ministère public ${ }^{15}$ ainsi que son corollaire, celui de la séparation des fonctions judiciaires sont des traits importants de notre système judicaire. Il est, dès lors, fondamental d'indiquer que cette indépendance est organique et fonctionnelle. Elle a été très clairement consacrée et exprimée, en droit congolais, dans le célèbre arrêt « Gecomines ${ }^{16}$ ».

De quoi avait-il été question? Dibunda Kabunji ${ }^{17}$ nous en donne les détails.

Dans cette affaire qui a opposé le ministère public à certains agents de la Gecomines ${ }^{18}$, la Cour d'Appel de Kinshasa avait, par son arrêt du 10 septembre 1968, ordonné au ministère public de procéder à un complément d'information dont les résultats ont été retenus par l'arrêt du13 mars 1969 pour condamner les prévenus dans cette cause.

La Cour Suprême avait constaté, par la suite, dans le fameux arrêt Gecomines que cet ordre donné au ministère public par la Cour d'appel n'avait aucun fondement dans la loi, car l'article 74 alinéa 6 CPP suivant lequel le tribunal saisi peut ordonner toute mesure d'instruction complémentaire nécessaire à la manifestation de la vérité, il ne peut être déduit que le tribunal a le droit d'enjoindre au ministère public de procéder à un complément d'information.

13 La Cour Suprême de justice.

14 LUZOLO ET BAYONA, op.cit.

15 Lire le sujet en droit français avec STEFANI GASTON, LEVASSEUR GEORGES et BOULOC BERNARD, Procédure pénale, Dalloz, Paris, 19eme Edition, 2004, ${ }^{\circ} 464$.

16 Bulletin des arrêts de la CSJ, vol n 1970 , fascicule I; P. 18.

17 DIBUNDA KABUNJI, «Les différentes violations de la loi constatées par la CSJ à l'occasion des pourvois en cassation », in Revue Juridique du Zä̈re, numéro spécial, 60eme année, P. 241 à 243.

18 Ancienne dénomination sociale de l'actuelle Gécamines. 
Cela avait obligé la Cour Suprême de Justice sous RP 2, 7 et 9 du 8 octobre 1969 (In RCB, 1970, I P. 18) à casser la partie de l'arrêt de la Cour d'appel du 10 septembre 1968 qui avait ordonné ce complément d'information, ainsi que conséquemment l'arrêt du 13 mars 1969 de la même Cour dans la mesure où celui-ci était la conséquence de la décision illégale du juge de fond.

La leçon retenue par le Doyen Nicolas-Abel Bayona à ce sujet, relativement à cette pratique tirée de la mauvaise lecture de l'article 74, alinéa 6 du Code de Procédure Pénale est digne d'intérêt dès lors que cet éminent professeur n'avait jamais cessé de rappeler à ses différents auditoires que la seule chose qu'ils se devaient de retenir désormais que jamais une juridiction ne peut ordonner au ministère public de compléter l'instruction dans tel ou tel autre sens.

\section{L'ARRET YAV MUKUTA KIBWE}

Le second arrêt de référence est celui que le professeur Luzolo Bambi et le même Doyen Bayona nous présentent dans leur ouvrage collectif ${ }^{19}$ et qu'ils commentent, d'ailleurs en ces termes :

" (...) les juges ne peuvent se permettre de blâmer les magistrats du parquet dans leurs décisions ou dans leurs paroles. Ils ne peuvent pas non plus donner des injonctions de poursuites aux magistrats du parquet. C'est dans ce sens que par arrêt du 02 juillet 1969 (Affaire MP contre Kinés Yav Mukuta Kiwe et Bindescedler), la cour Suprême de Justice a réaffirmé ce principe en cassant l'arrêt de la cour d'appel de Kinshasa, notamment au motif que cet arrêt a demandé au parquet de compléter l'enquête dans cette cause. ».

\section{LES RAISONS TIREES DE LA CONSTITUTION}

Le principe de la séparation des fonctions ${ }^{20}$, législative, judiciaire et exécutive, ainsi que son corollaire, dans le contexte judiciaire congolais, celui de la séparation des fonctions d'instruction et de poursuite, d'une part, et celui de jugement, d'autre part, trouvent leur consécration à partir de l'interprétation combinée, voire complémentaire, des articles 149, alinéa $1^{\mathrm{er}}$ et 151 , alinéa $1^{\mathrm{er}}$, de la constitution qui disposent, pour rappel, l'un et l'autre, respectivement :

"Le Pouvoir Judiciaire est indépendant du pouvoir législatif et du pouvoir exécutif. Il est dévolu aux cours et tribunaux qui sont : la cour constitutionnelle, la cour de cassation, le conseil d'Etat, la Haute Cour Militaire, les cours et tribunaux civils et militaires ainsi que les parquets rattachés à ces juridictions. (Article 149, alinéa 1 ${ }^{\text {er }}$ ).

19 LUZOLO BAMBI LESSA et BAYONA BA MEYA, op.cit.

20 LEVASSEUR GEORGES, Cours de Procédure Pénale, Paris, 1959. 
"Le pouvoir exécutif ne peut donner d'injonction au juge dans l'exercice de sa mission, et statuer sur les différends, ni entraver les cours de la justice, ni s'opposer à l'exercice d'une décision de justice (article 151, alinéa $1^{e r}$, de la constitution).

L'intérêt d'invoquer ces deux dispositions dans ce débat apparait, dès lors, plus nettement du fait que lesdites dispositions légales mettent suffisamment en évidence les conséquences de l'exclusion du ministère public du pouvoir judiciaire depuis la réforme constitutionnelle portée par la loi du 20 janvier 2011. En effet, bien que cela soit en réalité discutable, voire contestable, la constitution semble désormais considérer et le ministère public comme représentant et membre du pouvoir exécutif devant les cours et tribunaux.

C'est donc à ce titre - pose l'article 151 , alinéa $1^{\text {er }}$, de la constitution - ni le pouvoir exécutif, ni ses membres ne sont fondés de donner des injonctions ni au pouvoir judiciaire, ni aux membres de celui-ci. Cette interdiction vaut tout autant en sens contraire, ce qui nous conduit à conclure, du point de vue strictement judiciaire, que cette indépendance du Ministère Public à l'égard des juges ou des juridictions est réciproque.

Ce dernier argument constitue, même pour les moins réceptifs aux explications qui précèdent, le plus convainquant pour vrai que le juge congolais doit se considérer comme régulièrement affranchi ou délié de l'obligation d'appliquer une disposition légale contrainte à la constitution et ne saurait, dès lors, être reproché d'avoir appliqué une telle disposition d'essence supérieure à la loi (tels, le traité et la constitution) au détriment de la loi.

La jurisprudence des juridictions militaires recèle d'innombrables illustrations dans ce sens, par exemple, s'agissant des traités et accords internationaux, du recours au traité de Rome au détriment de la loi nationale s'agissant des crimes graves que vise celui-ci ou, encore, en droit interne, de l'application de l'article 19, alinéa 4, de la constitution, s'agissant des droits de tous de se faire assister, à tous les niveaux de la procédure, y compris l'enquête policière et l'instruction préparatoire, d'un défenseur de son choix et ce alors qu'aucune disposition de nos codes de procédure pénale, ni ordinaire, ni militaire, n'assure une telle garantie de procédure.

A vrai dire, ce principe constitutionnel de la séparation des fonctions judiciaires et celui de l'indépendance réciproques du ministère public et des juges, qui est son corollaire, conserve son sens et à sa raison d'être, celui d'éviter tout risque de despotisme et d'autocratie lorsqu'une seule autorité est investie des pouvoirs judiciaires exorbitants (notamment, celui d'instruire, de poursuivre ou de juger).

Il tend aussi à contenir soigneusement chaque autorité judiciaire dans son domaine légal d'intervention et dans les attributions spécifiques de ses fonctions et ce, lorsque même la loi en dispose autrement ou par une interprétation mécanique ou isolée de la loi l'on pense que c'est de la sorte que le législateur en a ainsi posé la règle.

Il interdit surtout, enfin, tel dans le cas d'espèce, aux juges de blâmer le Ministère Public, de lui faire des observations ou, encore moins, de lui donner des injonctions. Plusieurs conséquences procédurales sont attachées à la violation de ce principe d'indépendance réciproque sous étude, à savoir, sans nul doute, la censure de la Cour de cassation à lire la posi- 
tion de la Cour Suprême de Justice dans ses arrêts Gecomines et Yav sommairement commentés dans les lignes qui précèdent.

\section{CONCLUSION}

Cette étude aura certainement permis de réaliser que cette pratique, très courante chez les juges militaires, consistant, selon plusieurs formes, à faire des remontrances aux officiers du Ministère public, à les blâmer, à leur faire des observations sur telle ou telle autre question, notamment s'agissant tel ou tel autre aspect de leur saisine, voire - plus grave - à leur donner des injonctions, est simplement malheureuse.

Cette pratique décriée procède, on vient de le voir, d'une très mauvaise interprétation de la loi. Elle heurte et énerve, d'ailleurs, les dispositions pertinentes de la constitution, précisément ses articles 149 , alinéas $1^{\text {er }}$, et 151 , alinéas $1^{\text {er }}$, de la constitution qui ont très clairement posé, évidemment en règle constitutionnelle désormais, le principe de la séparation des fonctions judiciaires et, de manière encore plus lumineuse, l'interdiction incombant à l'un ou l'autre, dans le respect de leur indépendance réciproque, de se donner mutuellement des injonctions.

C'était aussi là l'occasion de décourager les interprétations mécaniques, journalistiques et isolées des dispositions légales, précisément de l'article 219, alinéa 2 CJM, car, on ne le dira jamais assez, l'interprétation d'un texte sans contexte, est un prétexte. C'est ainsi que par un raisonnement juridique normal, largement teinté de bon sens et qui a tenu compte des précédents historiques de cette disposition concernée, nous a permis, surtout avec l'éclairage des dispositions constitutionnelles citées ci-haut, nous permis d'en arriver à la conclusion qui sanctionne l'intitulé de la présente étude.

Il en découle qu'au lieu de donner des injonctions au ministère public pour compléter le dossier toutes les fois qu'elle estimera que celui lui soumis par le ministère public comporte des lacunes, le juge ou la juridiction se doit, plutôt, avant dire droit, d'ordonner toute mesure complémentaire (expertise, descente sur les lieux, production des pièces à conviction, ...) qui s'impose. Le juge dispose, dans ces conditions et pour ce faire, de trois possibilités procédurales : la première est celle que lui offre l'article 219 CJM dans la phase procédurale précédant l'ouverture des débats ou de l'audience tandis que les deux dernières, celles qui lui confèrent les articles 249 et 253 , alinéa 4 , du code judiciaire militaire.

En effet, si la loi l'a, certes, investi, au visa de l'article 219 CJM, d'ordonner avant l'ouverture des débats ou de la première audience de la juridiction concernée, des mesures d'instruction supplémentaire ou complémentaire, elle est loin de lui avoir donné les pouvoirs d'obtenir ce supplément d'instruction par le truchement du ministère public et, plus grave, en devant lui adresser des injonctions. En le faisant, un tel jugement expose, de façon certaine, son œuvre à la censure de la cour de cassation actuellement.

Par ailleurs, dès l'entame du procès, la première possibilité procédurale en présence est celle que l'article 249 CJM confère au président de la composition du tribunal. Il s'agit du pouvoir lui reconnu de faire apporter, par des mandats de comparution ou d'amener toute 
personne dont l'audition lui parait nécessaire. La seconde, en revanche, est celui qui est reconnu à la juridiction toute entière d'ordonner, avant dire droit, les mesures d'instruction complémentaire qu'elle désire, mais encore une fois en aucun cas au Ministère Public. Bref le juge lui seul peut faire venir par devant lui toutes personnes dont l'audition semble être indispensable dans l'éclatement de la vérité. De manière définitive nous retenons ce qui suit : le juge pénal Congolais, civil ou militaire, n'a pas le Droit, ni de blâmer le ministère public, ni de lui faire des observations et, encore moins, de lui donner des injonctions.

\section{BIBLIOGRAPHIES SELECTIVES}

1. BAYONA NICOLAS-ABEL, Réflexions sur la portée de l'article 67 CPP, in Le Plumitif, Collection

B. Kingudi, ${ }^{\circ} 4$, Lubumbashi, 2017. (qui a repris la version originaire de l'article de la Revue Congolaise de droit).

2. Bulletin des arrêts de la CSJ, vol n¹970, fascicule I.

3. DIBUNDA KABUNJI, «Les différentes violations de la loi constatées par la CSJ à l'occasion des pourvois en cassation », in Revue Juridique du Zaïre, numéro spécial, $60^{\mathrm{eme}}$ année.

4. Http ://www.coe.int/t/dghl/cooperation/CCPE

5. LEVASSEUR GEORGES, Cours de Procédure Pénale, Paris, 1959

6. LUZOLO BAMBI LESSA ET BAYONA BA MEYA, Manuel de Procédure Pénale, PUC, Kinshasa, 2011.

7. STEFANI GASTON, LEVASSEUR GEORGES et BOULOC BERNARD, Procédure pénale, Dalloz, Paris, $19^{\mathrm{eme}}$ Edition, 2004, $\mathrm{n}^{\circ} 464$.

8. VITU ANDRE, Procédure Pénale, PUF, Paris, 1957. 